\title{
Research on vibration monitoring and fault diagnosis of rotating machinery based on internet of things technology
}

https://doi.org/10.1515/nleng-2021-0019

Received Jun 22, 2021; accepted Jul 14, 2021.

\begin{abstract}
Recently, researchers are investing more fervently in fault diagnosis area of electrical machines. The users and manufacturers of these various efforts are strong to contain diagnostic features in software for improving reliability and scalability. Internet of Things (IoT) has grown immensely and contributing for the development of recent technological advancements in industries, medical and various environmental applications. It provides efficient processing power through cloud, and presents various new opportunities for industrial automation by implementing IoT and industrial wireless sensor networks. The process of regular monitoring enables early detection of machine faults and hence beneficial for Industrial automation by providing efficient process control. The performance of fault detection and its classification by implementing machine-learning algorithms highly dependent on the amount of features involved. The accuracy of classification will adversely affect by the dimensionality features increment. To address these problems, the proposed work presents the extraction of relevant features based on oriented sport vector machine (FO-SVM). The proposed algorithm is capable for extracting the most relevant feature set and hence presenting the accurate classification of faults accordingly. The extraction of most relevant features before the process of classification results in higher classification accuracy. Moreover it is observed that the lesser dimensionality of propose process consumes less time and more suitable for cloud. The experimental analysis based on the implementation of proposed approach provides and solution for the monitoring of machine condition and prediction of fault accurately based on cloud platform using industrial wireless sensor networks and IoT service.
\end{abstract}

Keywords: industrial wireless sensor networks (IWSNs), internet of things (IoT), support vector machine, fault diagnosis

\section{Introduction}

The background of fault diagnosis and its protection is as ancient as the machineries themselves. Initially, the manufacturers of machineries and their users relied on modest safety such as protection from overcurrent and overvoltage. This precaution ensures reliability and safety operation. However, with the increase in number of tasks makes the machinery more complex. Therefore, the improvement in diagnosing faults is foremost requirement. The fault diagnosis at its very initial stage becomes very important because the unwanted downtime of any machine can cause high financial loses. Industrial wireless sensor networks provide more flexible system for communication and transmitting the information towards the desired location. In the recent advancement among the field of wireless sensor networks and IoT-based continuous monitoring is highly recommended for industrial automation due to their enormous capability for detecting objects. The wireless sensor networks requires more attention in its quality of service parameters such as energy consumption, reliable transmission of information and cost for obtaining higher performances. The biggest challenge for the wireless sensor networks and IoT based application is to manage huge amount of sensed field data in real time basis.

In mechanical field the rotating machinery system is widely adopted in majority of the applications. In rotating machinery the relative motion among coupling surfaces, machinery components are easily susceptible for suffer-

Xiaoran Zhang, Zhengzhou Vocational University of Information and Technology, Zhengzhou 450046, China, E-mail: xrnzhng@gmail.com

Kantilal Pitambar Rane, Professor and Principal, KCEs COEM JALGAON, India

Ismail Kakaravada, Prasad V Potluri Siddhartha Institute of Technology, Kanuru, Vijayawada, India

*Corresponding Author: Mohammad Shabaz, Institute of Engineering and Technology, Chitkara University, Punjab, India, E-mail: bhatsab4@gmail.com 
ing damage. Fault detection and its diagnosis is therefore essential for maintaining the efficiency of machinery. The major challenge for maintaining the good health of machinery is the detection of fault and its patterns [1]. Various approaches have been designed for the diagnosis of fault in rotating machinery such as bearings, gearboxes and many others [2]. Venkatasubramanian et al. [3] presented the systematical review of fault detection and its diagnosis considering knowledge, model, signal-based hybrid and active methodologies. One of the most successful system consists of three main steps: fault symptoms determination, extraction of highly sensitive features and patterns classification. Fault symptoms includes measurement of vibration, analysis of thermal features, acoustic parameters and various of other processes have been considered as health indices for monitoring rotating systems. Considering all of these parameters, the signals from vibration sensor have proved as the most effective for monitoring the health of rotating system.

In recent years in the category of signal from vibration sensors various sensitive features for the detection of fault have been studied. In most of the system the extraction of features is performed in time, frequency and time-frequency domains [4]. Kebabsa et al. [5] have proposed scheme for the extraction of fault features in time domain by utilizing cyclostationarity as a pointer for diagnosing gears. One feature for the diagnosis was introduced by Feng et al. [6], for the monitoring of gearboxes at various time varying operating conditions. In frequency domain sometimes diagnosis of fault features are very sensitive. From the studies it is observed that the spectral kurtosis is highly recommended and most popular feature of fault in frequency domain [7]. Considering the frequency domain, spectral kurtosis is an optimal the modulation approach which was proposed for diagnosing the the bearing defects [8]. The feature extraction in time frequency domain have gained much attention in comparison with feature extraction in time and frequency domains for both academic and industrial applications. Fault sensitive features have been efficiently generated by implementing continuous wavelet transform (CWT) [9], wavelet packet transform (WPT) [10], comblet transform (CT) [11], discrete wavelet transform (DWT) [12], second generation wavelet transform and other tools of time frequency domain. Considering feature extraction in one domain, many of the researchers have been contributing for detecting the faults in machinery in different domains. Igba et al. [13], propose an approach for the examination of vibrational characteristics of gearboxes considering time and frequency domains.

On the basis of feature extraction of fault there exist various classifiers that can be used for distinguishing the health level from various patterns of faults. Oreski $e t$ al. [14] proposed study that presents multiple stage selection of features by implementing genetic algorithms for the diagnosis of gearboxes. One more study presents the fault diagnosis of gearboxes by implementing support vector machine and genetic algorithm. Bathaie et al. [15] has introduced a system by implementing dynamic neural network for the diagnosis of faults in gas turbine. An artificial neural network is implemented for the automatic diagnosis of faults through the vibration signals [16]. It is observed that the deep learning approaches has gained much attention in the community of classification. Tang et al. [17] proposed a deep learning-based approach for the classification of health state and to diagnose the failures.

This article is an attempt for the fault diagnosis in in rotating systems by presenting a feature learning approach. The purpose of this study is to use the statistical learning and to present the feature optimisation and its classification for improving the fault diagnosis in machinery. The misclassification in the diagnosis and the improper scheduling of maintenance are the major drawbacks for the conditional monitoring which requires new operating scenarios and termed as novelty detection. The existing machine learning algorithms like Support Vector Machine (SVM) provides pattern recognition and highly recommended for classification of data. On the other hand Support Vector Machine (SVM) provides high dimensionality e of features there by reducing the accuracy.

\section{Related work}

This section describes the recent work proposed for the industrial automation by implementing WSNs and IoT service.

With continuous enhancement in the field of computing and emerging $5 \mathrm{G}$ communication system application based on IoT, cloud computing, WSNs and huge data processing approaches for the maintenance prediction of mechanical equipments. These technologies entirely focused on the next level development for the industrial automation.

\subsection{Industrial wireless sensor networks based approaches}

Wireless communication protocols and different it strategies are registered with the rapid evolution in industrial wireless sensor networks. Hanzalek et al. [18] proposed 
methodology for avoiding collision and efficient scheduling using cluster tree industrial wireless sensor networks. Sheikh et al. [19] proposed an algorithm for the avoidance of hidden node which provides quality of service parameter by implementing multi-hop communication through mesh networks. Li et al. [20] present study which deals with the problem of treatment for effective energy by adopting ant colony optimization approach. Wanger et al. [21] discussed centralised and decentralized methods for the optimal configuration of wireless sensor networks respectively. Recently there exist many studies which describes the application of industrial wireless sensor network for performing the monitoring for electrical machines such as induction motors, fault diagnosis in pumps and pipelines, machine manufacturing, structural monitoring of health and smart grids.

Koutroulis et al. [22] introduces study based on the industrial wireless sensor networks system for the vibration data acquisition to monitor the rotation in power plants. The authors implement a data fusion approach for the judgement of similarity in time series and then fusion algorithm at task level is applied for deciding the strategy for data delivery. Their approach is focused for reducing the total bandwidth and power consumption, but their work only applicable for redundant data from same resources. Lee et al. [23] proposed a monitoring and fault diagnosis system for the health of induction motor on the basis of industrial wireless sensor networks. The extraction of features and its classification is carried out by the neural network classifier. The industrial application of their proposed approach is limited for the cases where it is required to produce all possible faults from tested machine at prior. Lu et al. [24] proposed an approach for the monitoring of remote energy using wireless sensor networks by exploring the diagnostic algorithm. Seshadrinath et al. [25] presents the detailed analysis for the fault diagnosis implementing industrial wireless sensor networks and the experimentation is verified using two step classified approach.

\subsection{Internet of things based approach}

Internet of things concept was first introduced by the community of radio frequency identification development. The concept of internet of things is very e popular because of its rapid growth among mobile devices, its capability in real time communication, data analytics and computing at cloud platform. The internet of things is a communication network where multiple physical things are embedded together such as smart devices, electronics, sensors and soft- wares which provides the capability for the collection of data from real world and it's sharing through internet [26]. India future the mobile-to-mobile market will dominate by providing variety of applications through this technology. In the proposed approach the communication among the monitor and the machinery is achieved through uh GPRS, the barriers of distance are eliminated and thereby the automation is achieved across any other part of the world [27].

Dong et al. [28] proposed a network for interfacing the sensor to iot platform and discuss the usage of CPLD (complex programming logic device). Kong et al. [29] presents the analysis of DPM (dynamic power management) considering internet-of-things scenario for monitoring the condition of the system.

\subsection{Fault diagnosis based methods}

With the evolution of smart industries, current processes of industries are transforming and adopting various smart devices for its automation. The most important task in the industrial processes is to maintain the required performance which may be affected by various kind of faults. For maintaining the high throughput from industrial processes, it is essential to adopt accurate, quick and effective fault detection and its diagnosis process for improving the performance of entire machinery and systems [30]. Considering various of the substantial advantages that can be achieved from the reduction in process and cost parameters at the same time providing the improved quality and productivity, fault detection and diagnosis system has gained much attention from the academicians and the industrial people as well [31]. There exist many theoretical and experimental studies for various fault detection approaches to diverse the industrial processes. The methods used for fault detection can be further classified as data driven, knowledge and model-based methods [32]. More specifically model based and data driven methods requires less efforts of modelling and process knowledge of interest, hence providing huge impact on industrial processes. The model based and data driven methods are widely adopted because of their simplicity and effectiveness for the process of fault detection and its diagnosis [33]. One of the major issue in current monitoring field is the online real time fault detection and diagnosis. Through the adoption of fault detection tool, it becomes possible to enhance the efficiency of process and safety by accurately predicting the abnormal symptoms at very early stage of process faults. Xenakis et al. [34] presents a study which discuss 
the fault detection models for distinguishing the fault status from normal status.

There are various methods for detecting faults in machinery. The motor windings and huge generators rated above $4.5 \mathrm{kV}$, partial discharge approach provides reliable results [35]. This process can also be achieved by portable test known as TGA-B. However, for motors working at low-voltage, stator fault diagnosis measures are yet to be proved. The method is suggested for detecting turnby-turn faults by analysing the machine component of axial flux by utilizing a big coil wound concentrically about the channel of the machine [36]. Kim et al. [37] presents the review of a recent work for the condition monitoring of machineries. The exhaustive review summarises that the behaviour and the characteristics of various faults in machines were identify and then analysed. Chauhan et al. [38] propose an approach on the basis of two types of methods for the fault diagnosis considering feature behaviour for the applications of conditional monitoring.

\section{Methodology}

Fault detection and its diagnosis is very important for the prevention of machines from further damage in industrial automation. Therefore, the real time monitoring of machines health it is essential for fault prediction, its diagnosis which leads to avoid the occurrence of fault in future. This section describes the implementation of the proposed system fourfold detection and prevention on the basis of real time online conditional monitoring systems. The methodology adopted for the detection of fault and its classification utilizing Industrial Wireless Sensor Networks and Internet of Things service is depicted in Figure 1. The proposed design of the system is comprised of three main working phases: Deployment of Industrial Wireless Sensor Networks, Monitoring station for diagnosing faults in machinery and IoT based cloud platform.

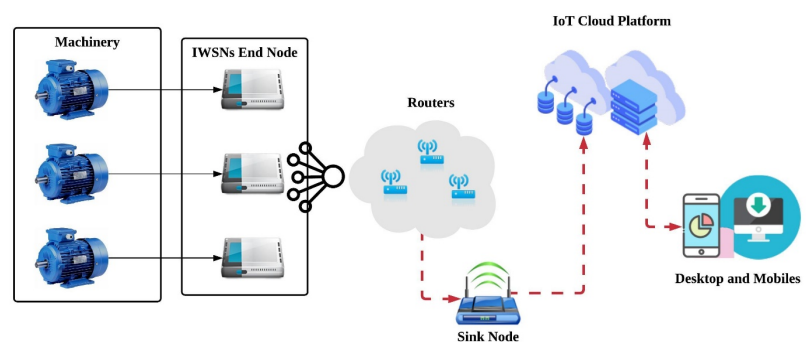

Figure 1: Proposed design of fault diagnosis system

\subsection{Deployment of IWSNs system}

The technologies of wireless communication have gained attention globally and very popular among the emerging applications for the Industrial automation. IEEE 803.15.4 wireless standard is adopted for the proposed system. The functional diagram of the proposed industrial wireless sensor networks system is depicted in Figure 2. The proposed system consists of four various sensors for measuring speed, vibration, voltage and current. Among all of these wireless sensor nodes basic node is deployed, which is implemented for handling the resource allocation, data fusion and channel scheduling considering the prior condition of data transmission. The implementation of functionality in the industrial wireless sensor networks helps preventing the redundant data and decreases transmission of large raw data, hence improving the sensor nodes lifetime. The data collected from multiple motor machines are transmitted to respective sink nodes, further transmits data packets to the coordinator node, which is located in the monitoring station. The coordinator node further interfaces with host system through serial port communication for diagnosing the identified faults at monitoring station.

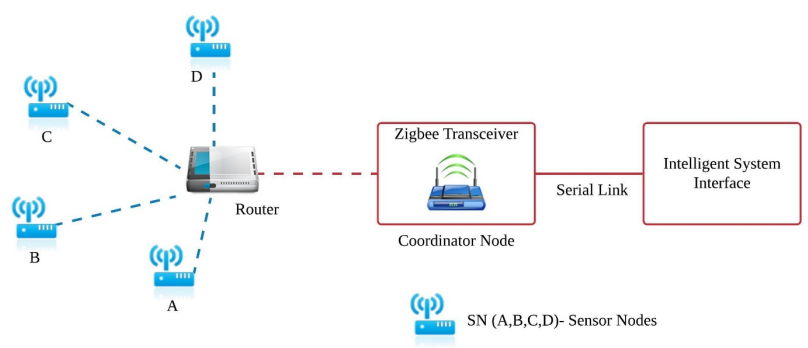

Figure 2: Functional structure of industrial wireless sensor networks

\subsection{Fault diagnosing approach in machinery}

The collected data from the deployed sensor nodes are processed in this system in order to diagnose the faults among machinery system. Due to the increment in number of features, fault detection and its classification is limited and therefore the feature set dimensionality is essential to be reduced. The proposed work presents the classification of faults through most relevant features by implementing Feature Oriented Support Vector Machine. Fault detection and its diagnosis process comprised of three various phases i.e., pre-processing, extraction of features and fault classification and diagnosis. 


\subsubsection{Data pre-processing phase}

The collected data set of speed, vibration, voltage and current analysed in the pre-processing phase. The collected data on the basis of electrical and mechanical analysis is processed on real time for fault detection. The process of data acquisition is carried out through industrial wireless sensor networks systems in which the weekend entries from real time collected data are removed through host system at pre-processing phase.

\subsubsection{Feature extraction phase}

The features are extracted in this phase in order to observe the fault nature which is either individual or combinational. The proposed fault-oriented sport vector machine system is analysed considering for various parameters of machinery under different scenarios of faults. In feature extraction phase list record for each of the class is processed and then the feature regarding minimum and maximum points are extracted. The maximum and minimum values of the features are extracted using this formula as follows in Equation 1:

$$
\begin{aligned}
& F T_{a}=\min \left(L_{V_{T}}\right) \text { and } F T_{b}=\max \left(L_{V_{T}}\right) \\
& F T_{c}=\min \left(L_{C_{T}}\right) \text { and } F T_{d}=\max \left(L_{C_{T}}\right) \\
& F T_{e}=\min \left(L_{R P M}\right) \text { and } F T_{f}=\max \left(L_{R P M}\right) \\
& F T_{g}=\min \left(L_{V i b}\right) \text { and } F T_{h}=\max \left(L_{V i b}\right)
\end{aligned}
$$

By computing these features, and their comparison the fault diagnosis classifies the fault.

\subsubsection{Fault classification phase}

The initialization of tested or classified data is observed as $T D S_{n}$. The tested values for various fault conditions in different categories such as vibration, speed, voltage and current are observed as $V i b_{T d}, R P M_{T d}, V_{T d}$ and $C_{T d}$. In the classification phase, Feature Oriented Support Vector Machine is applied for fault classification based on extracted relevant set of features.

The applied formula for the classification of relevant features is expressed in Equation 2:

$$
T D_{S C}=\min \frac{1}{2}\|\omega\|+C l_{N}(\text { analogous to fault })-\sum_{i: y_{i}} \tau_{i}
$$

Where, $\omega$ represents weight analogous to feature set, $C l_{N}$ represents class and $\tau_{i}$ represents the slack variable. The proposed approach utilizing most relevant feature for fault classification is described below:

\subsubsection{Classification approach}

Step 1: Initialization for size $X=1$ to $T D S_{n}$

Step 2: From size $X=1$ to $C l_{n}$

Step 3: If $\left(V_{T d} \geq F T_{a} \& \& V_{T d} \leq F T_{b}\right)$

If $\left(C_{T d} \geq F T_{c} \& \& C_{T d} \leq F T_{d}\right)$

If $\left(R P M \geq F T_{e} \& \& R P M \leq F T_{f}\right)$

If $\left(V i b_{T d} \geq F T_{g} \& \& V i b_{T d} \leq F T_{h}\right)$

Then,

$$
T D_{S C}=\min \frac{1}{2} \omega^{2}+C l_{N}(\text { analogous to fault })-\sum_{i: y_{i}} \tau_{i}
$$

Else, then $T D_{S C}=0$

End if;

End Y;

End $\mathrm{X}$;

\subsection{IoT based cloud analysis system}

As described in the previous section the observed performance from most relevant feature extraction for fault diagnosis system has achieved accuracy of $98.2 \%$ for fault classification. Fault oriented support vector machine algorithm is suitable for IoT based cloud platform for the prediction of faults in real time basis. Various state-of-art services are provided by the cloud vendors for the different IoT operations. Services such as remote control, analysis of data, machine learning and deep learning can be implemented based on specific application requirements through cloud platform.

\subsubsection{Instance creation through cloud platform}

ThingSpeak is an IoT based cloud platform, which is utilised for the implementation of present work for the storage and analysis of the data on real time. Instance creation of the required parameter in runtime environment through ThingSpeak cloud platform is the main foundation work of this present implementation, which is thereby utilised for the incorporation of Internet of Things and services of data storage and their interconnection. ThingSpeak also provides a programming tool based on cloud platform, which is linked, with the application environment on real time. 
The proposed FO-SVM for most relevant feature extraction to detect and diagnose faults is developed with python script in ThingSpeak IoT platform. The main function block in linked with IoT which continuously receives the data from the deployed sensors in real time. The data collected and received through the protocol is in JSON form as message. The functional block extract this message by implementing the python script and then fault prediction is initiated through the insistence of learning process.

\subsubsection{Internet of things and data storage implementation}

The service of internet of things bridges a communication link among the sensor nodes and real time environment application through cloud platform. In internet of things platform the interlinked devices communicate with each other by implementing lightweight protocol. The data communication is handled with the light weight protocol along with JSON message proposed work.

\section{Experimental scenario}

This section describes the experimental setup of proposed FO-SVM, for detection and diagnosis of machinery faults. The experimental scenario for the proposed system which includes IWSNs deployment and monitoring station is depicted in Figure 3. The specifications of machinery is tabulated in Table 1.

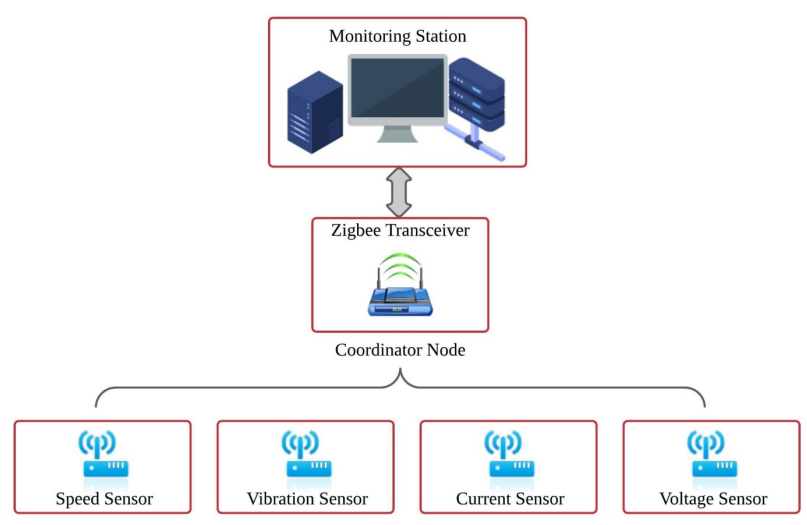

Figure 3: Experimental scenario for sensor and coordinator node

The fault diagnosis performance of proposed Fault Oriented Support Vector Machine Algorithm is depicted in Figure 4.
Table 1: Specifications of motor in experimentation

\begin{tabular}{l|l}
\hline Specification & Parameter value \\
\hline Category & III-phase motor \\
Voltage & 420 Volt $\pm 10 \%$ \\
Current & $2 \mathrm{~A}$ \\
Power & $0.40 \mathrm{~kW}$ \\
Power factor & 0.80 \\
Frequency & $55 \mathrm{~Hz} \pm 5 \%$ \\
Speed & $1425 \mathrm{RPM}$ \\
\hline
\end{tabular}

The experiments are conducted MATLAB simulation environment and its validation is carried out with real time fault data set of machinery specifications. The parameters of accuracy, precision and specificity are improved by the feature extraction of minimum and maximum ranges. The percentage improvement accuracy, precision and specificity are observed as $66.4 \%, 61.2 \%$ and $13 \%$ respectively in comparison with the support vector machine algorithm. The percentage improvement and its comparison are depicted in Figure 4( $a$ and $b)$. The observed percentage accuracy for classification of faults through proposed approach is $98.2 \%$.

\subsection{Sensor nodes and communication system}

Four different sensor nodes (speed, vibration, current and voltage) along with communication devices are utilized for the implementation of proposed work. One potential transformer (A $240 \mathrm{~V}$ ) consisting of a (TL082A) dual operational amplifier is deployed for measuring voltage. One (HE026T) hall effect sensor node is utilized for measuring the current in machinery. In order to measure the speed and vibration of the machinery, IP18455DF along with accelerometer (ADXL) is utilized. IEEE 802.15.4 (Zigbee) that offers $250 \mathrm{~kb} / \mathrm{s}$ of defined rate is implemented as wireless communication device.

\subsection{Experimental results}

Figure $5(a, b)$ represents the statistical features and waveform in time domain for the first signal received during experimental setup. As the receive signal creatures $0.41 \mathrm{sec}-$ onds at frequency of $10 \mathrm{kHz}$, the observed length of the discrete time signal is 4088 .

These vibrational signals are further transform to frequency domain. The statistical features along with the frequency domain is detected in Figure $6(\mathrm{a}, \mathrm{b})$. The observed 


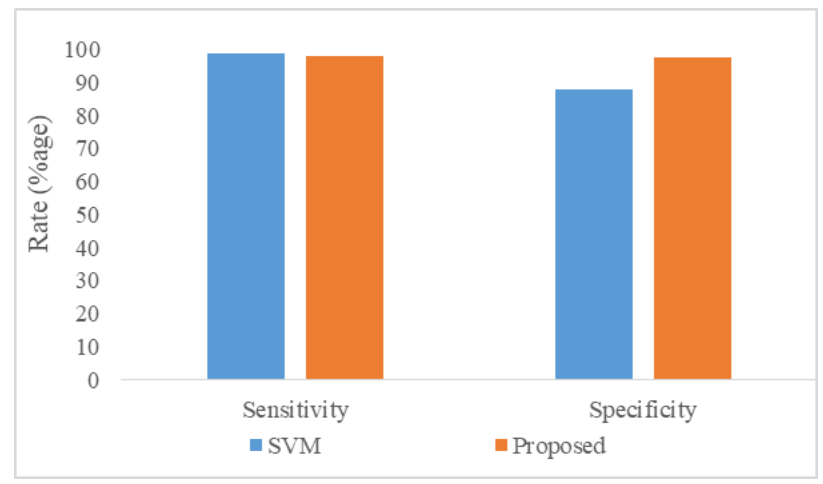

(a)

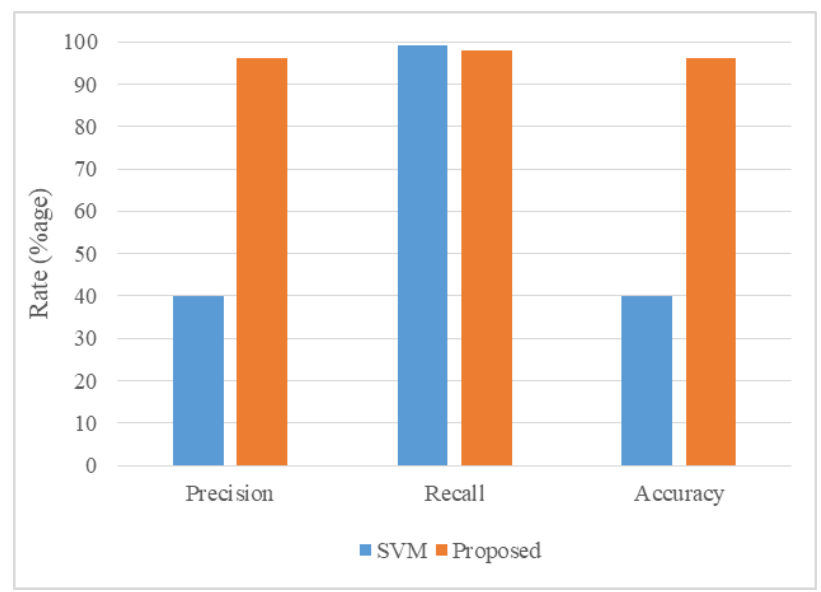

(b)

Figure 4: Performance indices; $(a, b)$ performance evaluation of fault diagnosis

frequency band at sampling frequency of $10 \mathrm{kHz}$ is 5000 Hz.

The road was further decomposed to four different levels for generating the time frequency domain signals. The statistical features and all four nodes together are depicted in Figure $7(\mathrm{a}, \mathrm{b})$. When the length of the row signal is 4088, observed data points at different for levels are 2045, 1026, 522 and 258, respectively. Therefore the number of observed data points as depicted in figure 7 (a) is 16,596.

A multiple class support vector machine classifier is then applied for obtaining first the diagnosis of fault. In the experimentation we have not considered learning models such as random forest, neural network and decision tree. this is because of the fact that support vector machine has more prominent representation and it performs better than these learning approaches. Fault diagnosis of the patients are tabulated in Table 2, and it is observed that the statistical features are higher (on average 73\%) from the different classification rates at time frequency domain in comparison with time and frequency domain. Among all of other

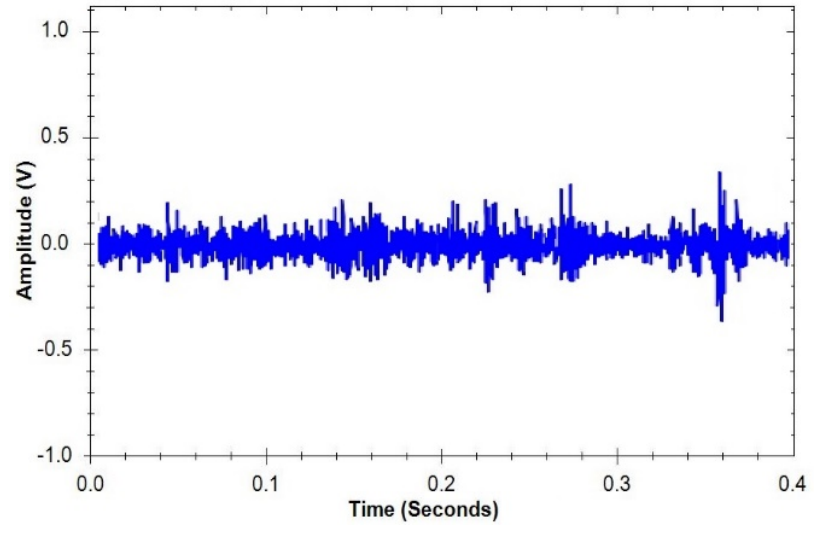

(a)

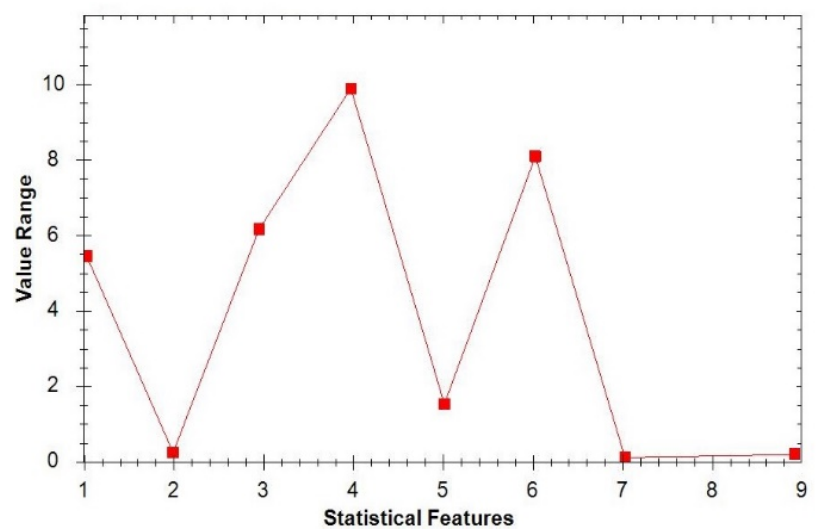

(b)

Figure 5: Feature analysis in time domain for fault diagnosis: (a) waveform in time domain of the first signal; (b) statistical features of first signal in time domain

models the learning of statistical features propose model presents better classification rate for similar data as 63\%, $92 \%, 96.04 \%$ and $84.3 \%$ respectively.

Figure $8(a, b)$ depicts the change in classification rate of fault and respect with the increase in in pre-training epochs for different features. For the diagnosis of fault in machinery the number of free training reports cannot much affect the classification. It is observed from the experimentation that for a few numbers of epochs the pretraining can attain good effect.

\section{Conclusion}

Fault detection and its diagnosis is an important process in order to prevent other damages of machines industries. In this work, a simple algorithm is designed for fault diagnosis in motor and machinery through IoT environment with Industrial Wireless Sensor Networks. The imple- 
Table 2: Classification rate of faults of dataset in various domains

\begin{tabular}{l|l|l|l|l|l}
\hline System & Domain & \multicolumn{4}{|l|}{ Diagnosis of faults } \\
\hline & & Decision tree & Random forest & $\begin{array}{l}\text { Neural net- } \\
\text { work }\end{array}$ & $\begin{array}{l}\text { Proposed } \\
\text { model }\end{array}$ \\
\hline Motor & Time & 27.26 & 61.25 & 36.28 & 63.25 \\
\hline & Frequency & 53.14 & 53.65 & 80.21 & 92.54 \\
\hline & Time-frequency & 46.02 & 70.24 & 79.65 & 96.74 \\
\hline Machinery & Time & 19.35 & 60.59 & 42.36 & 61.38 \\
\hline & Frequency & 40.16 & 81.21 & 83.47 & 88.65 \\
\hline & Time-frequency & 59.36 & 82.45 & 83.94 & 92.62 \\
\hline
\end{tabular}

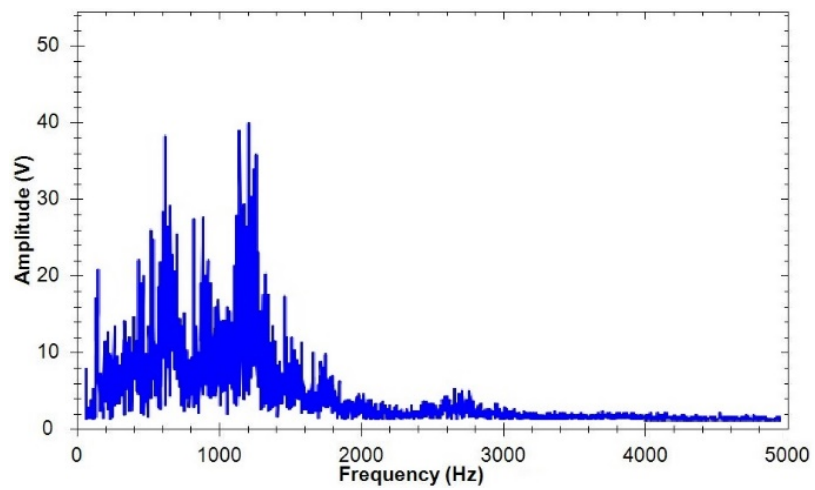

(a)

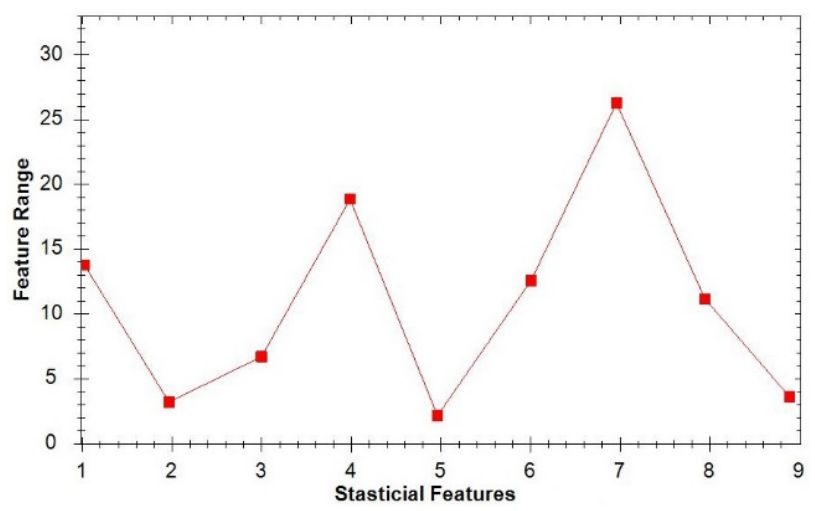

(b)

Figure 6: Feature analysis in frequency domain for fault diagnosis: (a) waveform in frequency domain of the first signal; (b) statistical features of first signal in frequency domain

mentation of sink node along with the coordinator node and various other sensor nodes improved the lifetime of the network and it also reduces the transmission delay through data fusion and efficient resource allocation. Along with the implementation of above communication system, diagnosis of fault is achieved with fault-oriented support vector machine algorithm. The performance evaluation of the present work reveals that the proposed algorithm improves the specificity precision and accuracy considering dimensionality reduction of suitable features.

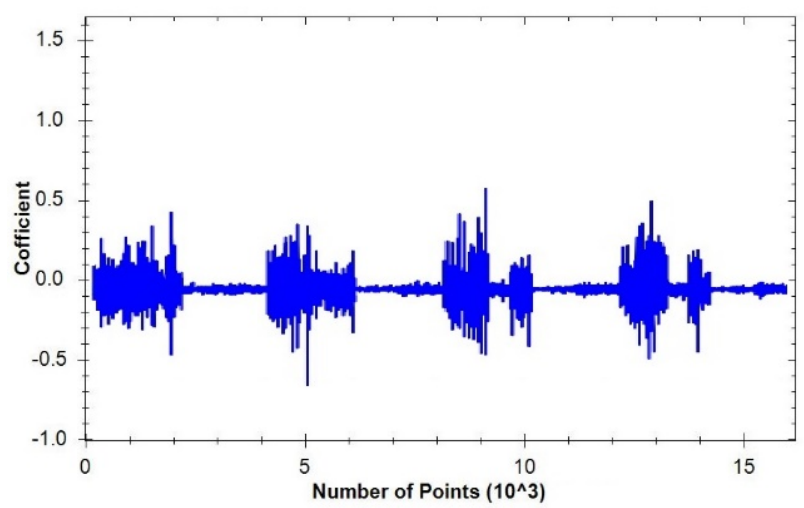

(a)

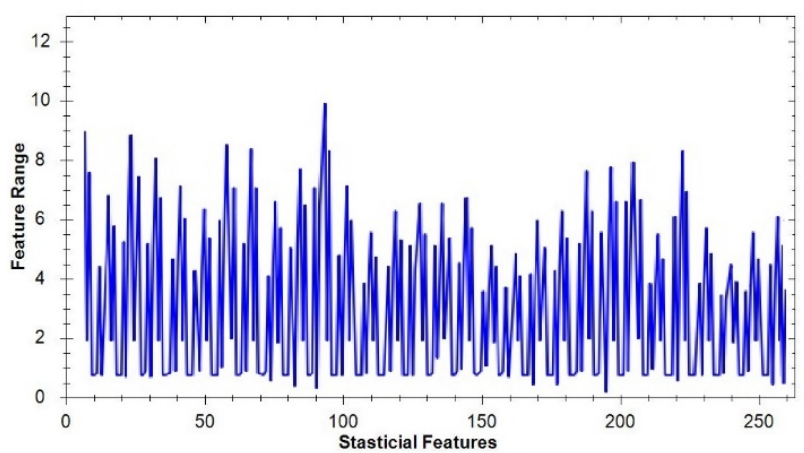

(b)

Figure 7: Feature analysis in time-frequency domain for fault diagnosis: (a) Waveform in time-frequency domain of the first signal; (b) Statistical features of first signal in time-frequency domain

Expected most relevant features are deployed to the ThingSpeak cloud platform for the protection of fault class. It is observed from the experimentation that different classes of faults are accurately predicted with less time interval. The entire structure of the proposed work ok improves the functionality for diagnosing faults in machine and hence suitable for the Industrial automation. The future work is motivated in identifying other methods for fault diagnosing on the basis of axial flux measurements, monitoring of current and voltage and vibration analysis, 


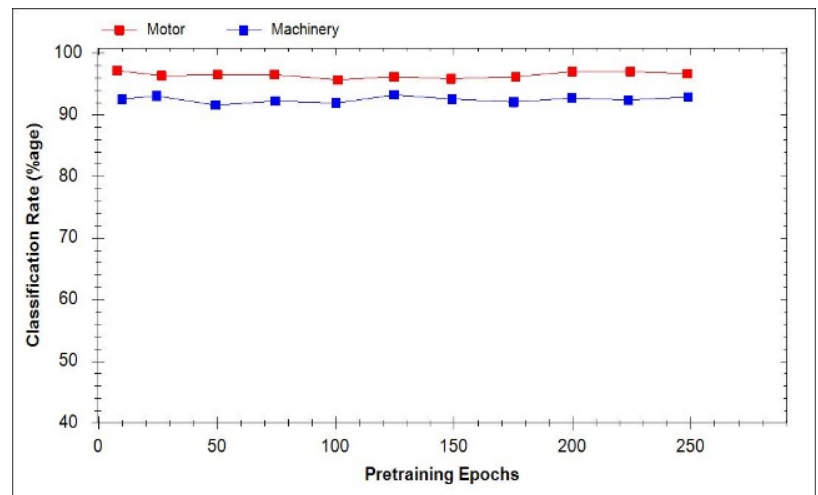

(a)

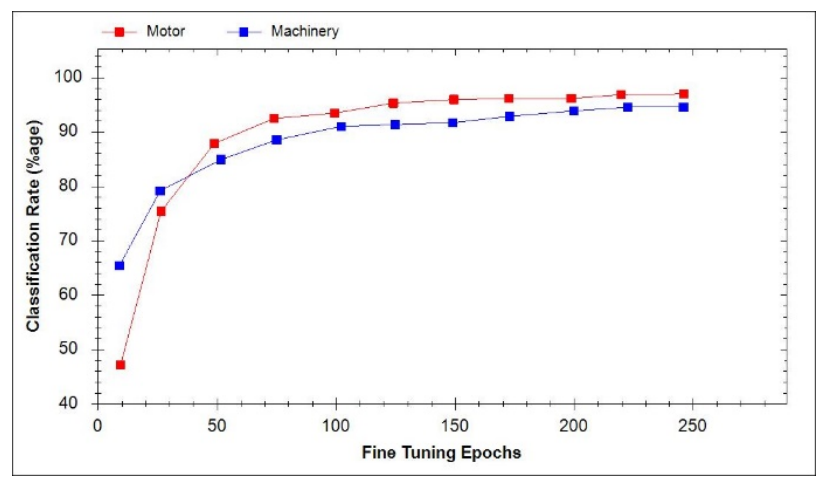

(b)

Figure 8: Relation among various epochs and classification rate: (a) classification rate and pretraining epochs; (b) classification rate and fine tuning epochs in time-frequency domain

etc. The future work includes the process of automated fault diagnosis for improving the design efficiency.

Funding information: The authors state no funding involved.

Author contributions: All authors have accepted responsibility for the entire content of this manuscript and approved its submission.

Conflict of interest: The authors state no conflict of interest.

\section{References}

[1] Kiangala KS, Wang Z. Initiating predictive maintenance for a conveyor motor in a bottling plant using industry 4.0 concepts. Int J Adv Manuf Technol. 2018 May 24;97(9-12):325171. Available from: http://dx.doi.org/10.1007/s00170-0182093-8
[2] Xia M, Li T, Xu L, Liu L, de Silva CW. Fault Diagnosis for Rotating Machinery Using Multiple Sensors and Convolutional Neural Networks. IEEE/ASME Trans Mechatron. 2018 Feb;23(1):101-10. Available from: http://dx.doi.org/10.1109/TMECH.2017.2728371

[3] Venkatasubramanian V, Rengaswamy R, Kavuri SN, Yin K. A review of process fault detection and diagnosis. Computers \& Chemical Engineering. 2003 Mar;27(3):327-46. Available from: http://dx.doi.org/10.1016/S0098-1354(02)00162-X

[4] Yang Y, Dong XJ, Peng ZK, Zhang WM, Meng G. Vibration signal analysis using parameterized time-frequency method for features extraction of varying-speed rotary machinery. Journal of Sound and Vibration. 2015 Jan;335:350-66. Available from: http://dx.doi.org/10.1016/j.jsv.2014.09.025

[5] Kebabsa T, Ouelaa N, Antoni J, Djamaa MC, Khettabi R, Djebala $A$. Experimental study of a turbo-alternator in industrial environment using cyclostationarity analysis. Int J Adv Manuf Technol. 2015 May 10;81(1-4):537-52. Available from: http://dx.doi.org/10.1007/s00170-015-7083-5

[6] Feng Y, Qiu Y, Crabtree CJ, Long H, Tavner PJ. Monitoring wind turbine gearboxes. Wind Energ. 2012 Jul 17;16(5):728-40. Available from: http://dx.doi.org/10.1002/we.1521

[7] Wang H, Yang G-H. A finite frequency domain approach to fault detection for linear discrete-time systems. Int J Control. 2008;81(7):1162-71. Available from: http://dx.doi.org/10.1080/00207170701691513

[8] Wang Y, Xiang J, Markert R, Liang M. Spectral kurtosis for fault detection, diagnosis and prognostics of rotating machines: A review with applications. Mechanical Systems and Signal Processing. 2016 Jan;66-67:679-98. Available from: http://dx.doi.org/10.1016/j.ymssp.2015.04.039

[9] Jawadekar A, Paraskar S, Jadhav S, Dhole G. Artificial neural network-based induction motor fault classifier using continuous wavelet transform. Systems Science \& Control Engineering. 2014 Nov 3;2(1):684-90. Available from: http://dx.doi.org/10.1080/21642583.2014.956266

[10] Saadaoui S, Tabaa M, Monteiro F, Chehaitly M, Dandache A. Discrete Wavelet Packet Transform-Based Industrial Digital Wireless Communication Systems. Information. 2019 Mar 7;10(3):104. Available from: http://dx.doi.org/10.3390/info10030104

[11] Wang D, Tse PW, Guo W, Miao Q. Support vector data description for fusion of multiple health indicators for enhancing gearbox fault diagnosis and prognosis. Meas Sci Technol. 2010 Dec 23;22(2):025102. Available from: http://dx.doi.org/10.1088/0957-0233/22/2/025102

[12] Abdulhady Jaber A, Bicker R. Fault Diagnosis of Industrial Robot Bearings Based on Discrete Wavelet Transform and Artificial Neural Network. IJPHM. 2020 Nov 11;7(2). Available from: http://dx.doi.org/10.36001/ijphm.2016.v7i2.2365

[13] Igba, J., Alemzadeh, K., Durugbo, C., \& Henningsen, K. (2015). Performance assessment of wind turbine gearboxes using in-service data: Current approaches and future trends. Renewable and Sustainable Energy Reviews, 50, 144-159. https://doi.org/10.1016/j.rser.2015.04.139

[14] Oreski S, Oreski G. Genetic algorithm-based heuristic for feature selection in credit risk assessment. Expert Systems with Applications. 2014 Mar;41(4):2052-64. Available from: http://dx.doi.org/10.1016/j.eswa.2013.09.004 
[15] Sina Tayarani-Bathaie S, Sadough Vanini ZN, Khorasani K. Dynamic neural network-based fault diagnosis of gas turbine engines. Neurocomputing. 2014 Feb;125:153-65. Available from: http://dx.doi.org/10.1016/j.neucom.2012.06.050

[16] Patan K, Witczak M, Korbicz J. Towards Robustness in Neural Network Based Fault Diagnosis. International Journal of Applied Mathematics and Computer Science. 2008 Dec 1;18(4):443-54. Available from: http://dx.doi.org/10.2478/v10006-008-0039-2

[17] Tang S, Yuan S, Zhu Y. Deep Learning-Based Intelligent Fault Diagnosis Methods Toward Rotating Machinery. IEEE Access. 2020;8:9335-46. Available from: http://dx.doi.org/10.1109/ACCESS.2019.2963092

[18] Hanzálek Z, Jurčík P. Energy Efficient Scheduling for Cluster-Tree Wireless Sensor Networks With Time-Bounded Data Flows: Application to IEEE 802.15.4/ZigBee. IEEE Trans Ind Inf. 2010 Aug;6(3):438-50. Available from: http://dx.doi.org/10.1109/TII.2010.2050144

[19] Sheikh SM, Wolhuter R, Engelbrecht HA. A survey of crosslayer protocols for IEEE 802.11 wireless multi-hop mesh networks. Int J Commun Syst. 2016 Apr 7;30(6):e3129. Available from: http://dx.doi.org/10.1002/dac.3129

[20] Li Q, Zhang C, Chen P, Yin YX. Improved ant colony optimization on particle swarm optimization. Control and Decis. 2013;28(6):873-878.

[21] Wagner, T., Hausner, C., Elger, J., Lowen, U., \& Luder, A. (2010). Engineering Processes for Decentralized Factory Automation Systems. In Factory Automation. InTech. https://doi.org/10.5772/9504

[22] Koutroulis, E., \& Kalaitzakis, K. (2003). Development of an integrated data-acquisition system for renewable energy sources systems monitoring. Renewable Energy, 28(1), 139152. https://doi.org/10.1016/s0960-1481(01)00197-5

[23] Lee, C.-Y., \& Wen, M.-S. (2020). Establish Induction Motor Fault Diagnosis System Based on Feature Selection Approaches with MRA. Processes, 8(9), 1055. https://doi.org/10.3390/pr8091055

[24] Bin Lu, \& Gungor, V. C. (2009). Online and Remote Motor Energy Monitoring and Fault Diagnostics Using Wireless Sensor Networks. IEEE Transactions on Industrial Electronics, 56(11), 4651-4659. https://doi.org/10.1109/tie.2009.2028349

[25] Seshadrinath, J., Singh, B., \& Panigrahi, B. K. (2014). Vibration Analysis Based Interturn Fault Diagnosis in Induction Machines. IEEE Transactions on Industrial Informatics, 10(1), 340-350. https://doi.org/10.1109/tii.2013.2271979

[26] Xu, X., Chen, T., \& Minami, M. (2012). Intelligent fault prediction system based on internet of things. Computers \& Mathematics with Applications, 64(5), 833-839. https://doi.org/10.1016/j.camwa.2011.12.049

[27] Wang, C., Vo, H. T., \& Ni, P. (2015, December). An loT Application for Fault Diagnosis and Prediction. 2015 IEEE International Conference on Data Science and Data Intensive Systems. 2015 IEEE International Conference on Data Science and Data Intensive Systems (DSDIS). https://doi.org/10.1109/dsdis.2015.97

[28] Dong, Y., Ren, H., Dong, J., \& Wang, L. (2015). Study on Wireless Network Communication in Stage Hydraulic Monitoring System Based on Internet of Things. Discrete Dynamics in Nature and Society, 2015, 1-9. https://doi.org/10.1155/2015/652183
[29] Kong, X., Xu, Y., Jiao, Z., Dong, D., Yuan, X., \& Li, S. (2020). Fault Location Technology for Power System Based on Information About the Power Internet of Things. IEEE Transactions on Industrial Informatics, 16(10), 6682-6692. https://doi.org/10.1109/tii.2019.2960440

[30] Rafiee, J., Arvani, F., Harifi, A., \& Sadeghi, M. H. (2007). Intelligent condition monitoring of a gearbox using artificial neural network. Mechanical Systems and Signal Processing, 21(4), 1746-1754. https://doi.org/10.1016/j.ymssp.2006.08.005

[31] Pinte, G., Devos, S., Stallaert, B., Symens, W., Swevers, J., \& Sas, P. (2010). A piezo-based bearing for the active structural acoustic control of rotating machinery. Journal of Sound and Vibration, 329(9), 1235-1253. https://doi.org/10.1016/j.jsv.2009.10.036

[32] Iqbal, R., Maniak, T., Doctor, F., \& Karyotis, C. (2019). Fault Detection and Isolation in Industrial Processes Using Deep Learning Approaches. IEEE Transactions on Industrial Informatics, 15(5), 3077-3084. https://doi.org/10.1109/tii.2019.2902274

[33] Scheffel, R. M., Fröhlich, A. A., \& Silvestri, M. (2021). Automated fault detection for additive manufacturing using vibration sensors. International Journal of Computer Integrated Manufacturing, 34(5), 500-514. https://doi.org/10.1080/0951192x.2021.1901316

[34] Xenakis, A., Karageorgos, A., Lallas, E., Chis, A. E., \& González-Vélez, H. (2019). Towards Distributed IoT/Cloud based Fault Detection and Maintenance in Industrial Automation. Procedia Computer Science, 151, 683-690. https://doi.org/10.1016/j.procs.2019.04.091

[35] Nandi S, Toliyat HA, Li X. Condition Monitoring and Fault Diagnosis of Electrical Motors-A Review. IEEE Trans On Energy Conversion. 2005 Dec;20(4):719-29. Available from: http://dx.doi.org/10.1109/TEC.2005.847955

[36] Jun Zhang, Rui-Xin Li, Pu Han, Dong-Feng Wang, Xi-Chao Yin. Wavelet packet feature extraction for vibration monitoring and fault diagnosis of turbo-generator. In: Proceedings of the 2003 International Conference on Machine Learning and Cybernetics (IEEE Cat No03EX693). IEEE; Available from: http://dx.doi.org/10.1109/ICMLC.2003.1264446

[37] Kim W, Katipamula S. A review of fault detection and diagnostics methods for building systems. Science and Technology for the Built Environment. 2017 Apr 27;24(1):3-21. Available from: http://dx.doi.org/10.1080/23744731.2017.1318008

[38] Chauhan V, Surgenor B. Fault detection and classification in automated assembly machines using machine vision. Int J Adv Manuf Technol. 2016 Oct 17;90(9-12):2491-512. Available from: http://dx.doi.org/10.1007/s00170-016-9581-5 